\title{
Optimal control of mirror pulses for cold-atom interferometry
}

\author{
Jack C. Saywell,,${ }^{1, *}$ Ilya Kuprov, ${ }^{2}$ David Goodwin, ${ }^{2}$ Max Carey, ${ }^{1}$ and Tim Freegarde ${ }^{1}$ \\ ${ }^{1}$ School of Physics \& Astronomy, University of Southampton, Highfield, Southampton, SO17 1BJ, United Kingdom \\ ${ }^{2}$ School of Chemistry, University of Southampton, Highfield, Southampton, SO17 1BJ, United Kingdom
}

(Received 13 April 2018; published 22 August 2018; corrected 12 March 2019)

\begin{abstract}
Atom matterwave interferometry requires mirror and beam splitter pulses that are robust to inhomogeneities in field intensity, magnetic environment, atom velocity, and Zeeman substate. We present theoretical results which show that pulse shapes determined using quantum control methods can significantly improve interferometer performance by allowing broader atom distributions, larger interferometer areas, and higher contrast. We have applied gradient ascent pulse engineering (GRAPE) to optimize the design of phase-modulated mirror pulses for a Mach-Zehnder light-pulse atom interferometer, with the aim of increasing fringe contrast when averaged over atoms with an experimentally relevant range of velocities, beam intensities, and Zeeman states. Pulses were found to be highly robust to variations in detuning and coupling strength and offer a clear improvement in robustness over the best established composite pulses. The peak mirror fidelity in a cloud of $\sim 80 \mu \mathrm{K}^{85} \mathrm{Rb}$ atoms is predicted to be improved by a factor of 2 compared with standard rectangular $\pi$ pulses.
\end{abstract}

DOI: 10.1103/PhysRevA.98.023625

\section{INTRODUCTION}

Emerging quantum technologies require the coherent manipulation of quantum states. For example, ultraprecise coldatom-based sensors such as gravimeters, accelerometers, magnetometers, and gyroscopes [1-4] use interactions with laser pulses to form the beam splitters and mirrors of matterwave interferometers [5], and these " $\pi / 2$ " and " $\pi$ " pulses must operate with high fidelity if the best sensitivity is to be achieved by using pulse sequences to increase the interferometer area [6] or maximize the entanglement [7].

The fidelity of quantum state manipulation deteriorates when there are inhomogeneities in the interaction field, magnetic environment, atomic velocities, and quantum state distributions [8]. This limits the number of control operations that can be performed before coherence is lost, so it is common to filter the atomic sample to restrict the variations experienced $[6,9,10]$ by fewer atoms. Inhomogeneities thus limit the interferometer area and sample size, and hence the measurement contrast and sensitivity [11,12].

Various techniques have been developed in the field of nuclear magnetic resonance (NMR) spectroscopy to produce control pulses that are robust to variations in the interaction strength and detuning, and such techniques should be applicable to other systems, including the effective two-level schemes of atom interferometry. Shaped pulses [13-15], rapid adiabatic pulses [16-18], and composite pulses [19-22] all use complex time-dependent interactions to reproduce the desired operation of a single pulse while compensating for the

\footnotetext{
*j.c.saywell@soton.ac.uk

Published by the American Physical Society under the terms of the Creative Commons Attribution 4.0 International license. Further distribution of this work must maintain attribution to the author(s) and the published article's title, journal citation, and DOI.
}

effects of inhomogeneities. For atom interferometry, McGuirk et al. [10] suggested that composite pulses could improve the augmentation pulses within large momentum transfer (LMT) arrangements, and Butts et al. [12] demonstrated that the WALTZ [23] composite inversion pulse doubled the sensitivity of a cold Cs atom interferometer.

In a feasibility study of the applicability of composite pulses to cold-atom interferometers, Dunning et al. [8] analyzed the performance of various established NMR pulse sequences for inversion or "mirror" operations in a thermal cloud of ${ }^{85} \mathrm{Rb}$ in a velocity-sensitive Raman arrangement subject to intensity variations and a distribution over Zeeman sublevels. Although cold-atom arrangements differ from NMR applications in the magnitudes of different inhomogeneities and the correlations between them, most of the schemes tested improved the robustness of the inversion operation to both detuning and coupling strength variations, and there was excellent agreement between the observed performance and that predicted computationally, showing there to be no further significant perturbations or decoherence.

In this paper, we address the computational design and optimization of mirror pulses specifically for atom interferometry. Using the well-known optimal control algorithm GRAPE [24] and the advanced spin dynamics simulation toolbox Spinach [25], we have derived pulse shapes that we predict will improve interferometric contrast following a Mach-Zehnder sequence within a $100 \mu \mathrm{K}$ cloud of ${ }^{85} \mathrm{Rb}$ atoms by a factor of 1.76 compared with standard rectangular pulses of constant phase and intensity. Our pulse shapes compensate off-resonance and pulse-length errors better than established composite pulse sequences, doubling the peak mirror fidelity when simulated in the $\sigma^{+}-\sigma^{+}$Raman polarization arrangement for a $\sim 80 \mu \mathrm{K}$ cloud. It is expected that such optimal pulses should allow for greater interferometric areas, higher contrast, warmer samples, and therefore increased interferometric sensitivities. 


\section{COHERENT PULSE THEORY}

\section{A. Two-level representation}

The Hamiltonian of a two-level atom interacting with a laser can be written in the rotating frame as [5]

$$
\hat{H}_{R}=\frac{\hbar}{2}\left(\begin{array}{cc}
\delta & \Omega_{R} e^{-i \phi_{L}} \\
\Omega_{R} e^{i \phi_{L}} & -\delta
\end{array}\right) .
$$

Here $\Omega_{R}$ is the Rabi frequency, $\phi_{L}$ is the laser phase, and $\delta(t)$ is the detuning from resonance. The detuning can be expressed as [5]

$$
\delta(t)=-\delta^{\mathrm{AC}}+\omega_{L}(t)-\left(\omega_{12}+\frac{\mathbf{p} \cdot \mathbf{k}_{L}}{m}+\frac{\hbar\left|\mathbf{k}_{L}\right|^{2}}{2 m}\right) .
$$

$\delta^{\mathrm{AC}}$ represents the ac Stark shift, $\omega_{L}(t)$ is the laser frequency, and $\omega_{12}$ is the separation of the two levels. $\mathbf{k}_{L}$ is the laser wave vector, $\hbar\left|\mathbf{k}_{L}\right|^{2} / 2 m$ is the recoil shift, and $\mathbf{p} \cdot \mathbf{k}_{L} / m$ is the Doppler shift. The two-level system hence consists of the states $|1, \mathbf{p}\rangle$ and $\left|2, \mathbf{p}+\hbar \mathbf{k}_{L}\right\rangle$, where 1 and 2 refer to the two atomic states and $\hbar \mathbf{k}_{L}$ is the photon impulse. If the beams are kept resonant at the center of the velocity distribution, e.g., by chirping the frequency to account for acceleration under gravity [1,14], the detuning can be written as $\left|\mathbf{k}_{L}\right| v$, where $v$ is the relative speed of a given atom along the direction of the wave vector. Therefore, the detuning of a given atom will remain approximately fixed throughout the interferometer sequence, and the range of detunings will be due to the momentum distribution and hence temperature of the cloud.

In the Bloch sphere representation, the state vector of any two-level system can be written as [26]

$$
|\psi\rangle=\cos \left(\frac{\vartheta}{2}\right)|1\rangle+e^{i \varphi} \sin \left(\frac{\vartheta}{2}\right)|2\rangle,
$$

where $\vartheta$ and $\varphi$ are the polar and azimuthal coordinates of a point on the surface of a unit sphere. Free and driven evolution is expressed in terms of rotations about axes through the center of the Bloch sphere. The propagator,

$$
\hat{U}\left(t_{1}, t_{2}\right)=\hat{T} \exp \left[-\frac{i}{\hbar} \int_{t_{1}}^{t_{2}} \hat{H}\left(t^{\prime}\right) d t^{\prime}\right],
$$

where $\hat{T}$ is the time-ordering operator [27], describes the unitary evolution of the quantum state $|\psi\rangle$ from time $t_{1}$ to $t_{2}$. Taking the matrix exponential of the Hamiltonian in the rotating frame [Eq. (1)], it can be shown that [28]

$$
\hat{U}(t, t+d t)=\exp \left[-\frac{i \boldsymbol{\Omega}(t) \cdot \boldsymbol{\sigma}}{2} d t\right],
$$

where $\sigma$ is the vector of Pauli matrices. Therefore, the effect of a pulse of duration $d t$ is to rotate the state on the surface of the Bloch sphere by an angle of magnitude

$$
\theta \equiv \tilde{\Omega}_{R}(t) d t=\sqrt{\Omega_{R}^{2}+\delta^{2}} d t
$$

about an axis defined by the field vector $\boldsymbol{\Omega}(t)$ :

$$
\boldsymbol{\Omega}=\Omega_{R} \cos \left(\phi_{L}\right) \mathbf{x}+\Omega_{R} \sin \left(\phi_{L}\right) \mathbf{y}+(\delta) \mathbf{z} .
$$

Recalling the identity

$$
\exp (i \alpha \hat{\mathbf{n}} \cdot \boldsymbol{\sigma})=\mathbf{I} \cos (\alpha)+i \hat{\mathbf{n}} \cdot \boldsymbol{\sigma} \sin (\alpha),
$$

the form of the propagator for a pulse with a constant field vector and duration $\Delta t$ is given by $[14,28]$

$$
\hat{U}=\left(\begin{array}{cc}
C^{*} & -i S^{*} \\
-i S & C
\end{array}\right)
$$

where $C$ and $S$ are defined as

$$
\begin{aligned}
C & \equiv \cos \left(\tilde{\Omega}_{R} \Delta t / 2\right)+i\left(\delta / \tilde{\Omega}_{R}\right) \sin \left(\tilde{\Omega}_{R} \Delta t / 2\right), \\
S & \equiv e^{i \phi_{L}}\left(\Omega_{R} / \tilde{\Omega}_{R}\right) \sin \left(\tilde{\Omega}_{R} \Delta t / 2\right) .
\end{aligned}
$$

\section{B. Inhomogeneities and composite pulses}

It is common in NMR spectroscopy to refer to off-resonance and pulse-length errors. An off-resonance error arises when the detuning $\delta$ is nonzero and the field vector does not lie perfectly in the $\vartheta=\pi / 2$ plane of the Bloch sphere. A pulse-length error occurs when the desired total rotation angle around the field vector is incorrect, due to either an error in the pulse duration or an error in the effective coupling strength $\tilde{\Omega}_{R}$. Detunings lead to deflection of the atomic state trajectory, and variations in the coupling strength or Rabi frequency lead to errors in the rotation angle around the field vector. These errors lead to dephasing and reductions in the fidelity of state manipulation [8].

Composite pulses are pulse sequences intended to replace a desired operation while providing robustness to off-resonance and pulse-length errors. Composite pulses compensate for such errors by using concatenated sequences with tailored phases and durations [19], which are equivalent to series of rotations on the Bloch sphere; both the combined laser phase $\left(\phi_{L}\right)$ and pulse duration $(\Delta t)$ may be different for each pulse in the sequence. The notation $\theta_{\phi}$ is used to specify a particular rotation element, so that a composite pulse can be written as the sequence

$$
\theta_{\phi_{1}}^{(1)} \theta_{\phi_{2}}^{(2)} \theta_{\phi_{3}}^{(3)} \cdots
$$

Such pulses can be placed into two categories: "point-to-point" (PP) rotations, and "universal rotations" (URs). The first are designed to only perform the correct rotations for specific starting states, whereas UR pulses are intended to perform the correct rotation for all starting points. The type of pulse required depends on the role in the application.

Although numerous composite pulses have been developed for NMR applications, most result from a combination of calculation and intuition, and there is no way to design or tailor a sequence for a particular application automatically. Numerical optimization of a control system has become a promising alternative for broadband pulse generation in NMR [29] and has been successfully applied to the control of Bose-Einstein condensates [30-32], the stabilization of ultracold molecules [33], the optimization of magneto-optical traps [34], and nitrogen-vacancy center magnetometry [35]. Optimal control theory offers methods to generate optimized pulse sequences which are tailored to specific systems and applications.

\section{OPTIMAL CONTROL THEORY}

Optimal control theory aims to obtain control parameters that allow a dynamical system to be driven so as to maximize 
some objective function. In quantum mechanics, this objective function often represents the accuracy with which initial states may be driven to desired final states by the available control fields, subject to constraints on the capabilities of the instruments such as maximum power and frequency.

The total Hamiltonian of a system can be written in the form

$$
\hat{H}(t)=\hat{H}^{(0)}+\sum_{n=1}^{M} c^{(n)}(t) \hat{H}^{(n)},
$$

where the drift Hamiltonian $\hat{H}^{(0)}$ represents the free evolution of the system. The operators $\hat{H}^{(n)}$ correspond to the experimental control fields whose amplitudes $c^{(n)}(t)$ can be set to form a given composite pulse sequence.

To determine the performance of a given pulse, we consider an example fidelity measure of the form

$$
\mathcal{F}\left\{c^{(n)}(t)\right\}=f\{\left\langle\psi_{D}\right| \underbrace{\hat{T}\left[\exp \int_{0}^{t_{f}}-\frac{i}{\hbar} \hat{H}(t) d t\right]\left|\psi_{0}\right\rangle}_{\text {resultant state }}\},
$$

where $\left|\psi_{D}\right\rangle$ is the desired target state, $\left|\psi_{0}\right\rangle$ is the initial state, and $f$ is a differentiable function of the projection of the resultant state onto $\left|\psi_{D}\right\rangle$. The aim is to either minimize or maximize $\mathcal{F}$ for all members of an ensemble with varying drifts. The choice of $\left|\psi_{0}\right\rangle,\left|\psi_{D}\right\rangle$, and $f$ is application dependent.

A well-known gradient-based optimization method, first developed by Khaneja et al. [24] for the design of NMR pulse sequences, is gradient ascent pulse engineering (GRAPE). GRAPE has computationally efficient gradients using analytical derivatives [36,37], which can be used to approximate and even compute the Hessian [38] and allow for Newton-Raphson-type optimizations.

GRAPE begins by discretizing the $\mathrm{M}$ control sequences $c^{(n)}(t)$ into $\mathrm{N}$ time steps $c_{k}^{(n)}$ of duration $\Delta t$ and assuming that during each time step the control amplitude $c_{k}^{(n)}$ is a constant. The optimization involves finding the vectors $\left\{c_{k}^{(n)}\right\}$ such that the chosen functional $\mathcal{F}$ is either maximal or minimal. The form of the time-ordered propagator is then simplified to a product of $k$ "slices":

$$
\hat{U}=\prod_{k} \exp \left[-\frac{i}{\hbar}\left(\hat{H}^{(0)}+\sum_{n=1}^{M} c_{k}^{(n)} \hat{H}^{(n)}\right) \Delta t_{k}\right] \equiv \prod_{k} \hat{U}_{k} .
$$

Therefore, $\mathcal{F}$ becomes

$$
\mathcal{F}=f\left(\left\langle\psi_{D}\left|\hat{U}_{N} \hat{U}_{N-1} \cdots \hat{U}_{k} \cdots \hat{U}_{1} \hat{U}_{0}\right| \psi_{0}\right\rangle\right) .
$$

For the optimization, derivatives of $\mathcal{F}$ are required with respect to the set of control coefficients $\left\{c_{k}^{(n)}\right\}$. GRAPE computes these derivatives in an efficient way, which can be seen by making the observation that the only element in Eq. (15) that depends on $c_{k}^{(n)}$ is $\hat{U}_{k}$. Therefore, computation of $\partial \mathcal{F} / \partial c_{k}^{(n)}$ for all time steps $k$ and all control channels $n$ requires just two simulations:

$$
\begin{aligned}
& \frac{\partial}{\partial c_{k}^{(n)}}\left(\left\langle\psi_{D}\left|\hat{U}_{N} \hat{U}_{N-1} \cdots \hat{U}_{k} \cdots \hat{U}_{1} \hat{U}_{0}\right| \psi_{0}\right\rangle\right) \\
& =\underbrace{\left\langle\psi_{D}\right| \hat{U}_{N} \hat{U}_{N-1} \cdots \hat{U}_{k+1}}_{\text {backward propagation }} \frac{\partial \hat{U}_{k}}{\partial c_{k}^{(n)}} \overbrace{\hat{U}_{k-1} \cdots \hat{U}_{1} \hat{U}_{0}\left|\psi_{0}\right\rangle}^{\text {forward propagation }} .
\end{aligned}
$$

The directional derivatives which require computation are

$$
\frac{\partial}{\partial c_{k}^{(n)}} \exp [-\frac{i}{\hbar} \underbrace{\left(\hat{H}^{(0)}+\sum_{n=1}^{M} c^{(n)}\left(t_{k}\right) \hat{H}^{(n)}\right)}_{\hat{H}_{k}} \Delta t] .
$$

An approximate expression for this derivative is given by Khaneja et al. [24]. First-order gradient ascent can then be used to optimize the fidelity $\mathcal{F}$ iteratively. Improvements were made in the use of propagator derivatives by De Fouquieres et al. [39], which use the gradient to approximate a second-order optimization method, called a quasi-Newton optimization, improving optimization convergence. This work uses the limited-memory Broyden-Fletcher-Goldfarb-Shanno quasi-Newton method implemented in Spinach [38,39] with analytical directional derivatives [37].

The Hamiltonian of our system [Eq. (1)] can be expressed as

$$
\hat{H}_{R}=\frac{\delta}{2} \hat{\sigma}_{z}+\frac{1}{2} \Omega_{R}(t)\left\{\cos \left[\phi_{L}(t)\right] \hat{\sigma}_{x}+\sin \left[\phi_{L}(t)\right] \hat{\sigma}_{y}\right\},
$$

where the drift Hamiltonian is $\hat{H}^{(0)}=(\delta / 2) \hat{\sigma}_{z}$ and the two control operators $\hat{H}^{(1)}$ and $\hat{H}^{(2)}$ can be identified with Pauli matrices $\hat{\sigma_{x}}$ and $\hat{\sigma_{y}}$, respectively. The two control coefficients $c^{(1)}(t), c^{(2)}(t)$ are given by $\Omega_{R}(t) \cos \left[\phi_{L}(t)\right]$ and $\Omega_{R}(t) \sin \left[\phi_{L}(t)\right]$, respectively. This form is directly analogous to the case of a spin system interacting with an applied rf field given appropriate parameter scaling of the magnitudes of each term [40].

Optimal control can be used to obtain optimal waveforms $\Omega_{R}(t)$ and $\phi_{L}(t)$, or the amplitude may be fixed and an optimal phase profile obtained. Initial guesses for the pulse waveform $\left[\Omega_{R}(t)\right.$ and $\left.\phi_{L}(t)\right]$ are provided, and the derivatives of the relevant fidelity measure are calculated, returned, and fed into the optimization module of Spinach [38]. The control pulses are made robust to variations in detuning (off-resonance errors) by providing Spinach with an ensemble of drift Hamiltonians and maximizing or minimizing the average of individual fidelities. Robustness to variations in power (pulse-length errors) is achieved by providing a range of power levels, which are averaged over in the fidelity calculation [41,42]. We define the ensemble provided to the optimization as consisting of a number of offsets in detuning and coupling strength, denoted by $\delta^{\text {off }}$ and $\Omega_{R}^{\text {off }}$, respectively. Finally, penalties can be added to the objective function to restrict experimentally relevant quantities such as maximum power or enforce waveform smoothness $[24,38]$. 


\section{INTERFEROMETER FIDELITY}

Although mirror pulses can be optimized individually, we obtain measures of mirror pulse fidelity by considering the role of the mirror within a three-pulse Mach-Zehnder sequence. In addition to the ability of a mirror pulse to transfer as many atoms from ground to excited state as possible, variations in the atomic phase of the final state following a mirror pulse must be minimized to preserve interferometric contrast, as shown by Luo et al. [14]. The excited state population after a sequential application of pulse propagators corresponding to the $\pi / 2-$ $\pi-\pi / 2$ sequence acting on an atom initially in the ground state can be obtained by following the analytical treatment given by Stoner et al. [28],

$$
\begin{aligned}
P_{2}= & \left|S_{\frac{\pi}{2}}\right|^{4}\left|S_{\pi}\right|^{2}+\left|C_{\frac{\pi}{2}}\right|^{4}\left|S_{\pi}\right|^{2}+2\left|S_{\frac{\pi}{2}}\right|^{2}\left|C_{\pi}\right|^{2}\left|C_{\frac{\pi}{2}}\right|^{2} \\
& -2 \operatorname{Re}\left[\exp \left(i \phi_{\mathrm{i}}\right) C_{\frac{\pi}{2}} S_{\frac{\pi}{2}}\left(S_{\pi}^{*}\right)^{2} C_{\frac{\pi}{2}}^{*} S_{\frac{\pi}{2}}\right],
\end{aligned}
$$

where $\mathrm{C}$ and $\mathrm{S}$ are defined in Eq. (10) and refer to elements of the pulse propagators. We have assumed the initial and final beam splitters in the Mach-Zehnder sequence to be identical and the dwell times between pulses to be the same. The subscripts " $\frac{\pi}{2}$ " and " $\pi$ " refer to the beam splitters and mirrors, respectively. $\phi_{\mathrm{i}}$ is an interferometric phase term which gives information about the inertial forces acting during the sequence, but which is modified by pulse-dependent phase shifts. This phase must be preserved when averaging over an ensemble of atoms with a distribution of velocities and coupling strengths. The interferometer output can be written as [14]

$$
P_{2}=\frac{1}{2}\left\{A(\delta)-B(\delta) \cos \left[\phi_{\mathrm{i}}+\phi_{\mathrm{p}}(\delta)\right]\right\},
$$

where $\phi_{\mathrm{p}}(\delta)$ is a phase shift introduced by the pulses and $A(\delta)$ and $B(\delta)$ represent the interferogram offset and contrast, respectively. In the ideal case $A(\delta), B(\delta)$, and $\phi_{\mathrm{p}}(\delta)$ should be constant for all detunings present in an atomic sample. For maximum contrast following thermal averaging, $A$ and $B$ must be unity for all atoms. These requirements can be used to obtain a measure of fidelity for the mirror pulse, which we can use to numerically optimize pulse shapes with these properties across an ensemble. The phase shift due to the pulses can be expanded as

$$
\begin{aligned}
\phi_{\mathrm{p}}(\delta) & =2 \phi S_{\frac{\pi}{2}}-2 \phi S_{\pi} \\
& =2 \phi\left(i\left\langle 2\left|\hat{U}_{\frac{\pi}{2}}\right| 1\right\rangle\right)-2 \phi\left(i\left\langle 2\left|\hat{U}_{\pi}\right| 1\right\rangle\right),
\end{aligned}
$$

and the contrast can be written as

$$
\begin{aligned}
B(\delta) & =4\left|C_{\frac{\pi}{2}}\right|^{2}\left|S_{\pi}\right|^{2}\left|S_{\frac{\pi}{2}}\right|^{2} \\
& =4\left|\left\langle 1\left|\hat{U}_{\frac{\pi}{2}}\right| 2\right\rangle\right|^{2}\left|\left\langle 1\left|\hat{U}_{\pi}\right| 2\right\rangle\right|^{2}\left|\left\langle 1\left|\hat{U}_{\frac{\pi}{2}}\right| 1\right\rangle\right|^{2} .
\end{aligned}
$$

Assuming rectangular and perfectly resonant beam-splitter pulses across the atomic ensemble $\left(\left|C_{\frac{\pi}{2}}\right|^{2}\left|S_{\frac{\pi}{2}}\right|^{2} \rightarrow 1 / 4\right) \mathrm{im}-$ plies that the following constraints on the mirror pulse for an ensemble of atoms will maximize contrast of the MachZehnder output after thermal averaging:

$$
\hat{U}_{\pi}\left\{\begin{array}{l}
\left|\left\langle 1\left|\hat{U}_{\pi}\right| 1\right\rangle\right|^{2}=0 \\
\left|\left\langle 2\left|\hat{U}_{\pi}\right| 1\right\rangle\right|^{2}=1 \\
\phi\left(\left\langle 2\left|\hat{U}_{\pi}\right| 1\right\rangle\right) \text { const. }
\end{array}\right.
$$

We therefore consider the following two mirror pulse fidelities:

$$
\begin{gathered}
\mathcal{F}_{\text {real }}^{\pi}=\operatorname{Re}\left\langle 2\left|\hat{U}_{\pi}\right| 1\right\rangle, \\
\mathcal{F}_{\text {imag }}^{\pi}=\operatorname{Im}\left\langle 2\left|\hat{U}_{\pi}\right| 1\right\rangle .
\end{gathered}
$$

Maximizing fidelity $\mathcal{F}_{\text {real }}^{\pi}$ or $\mathcal{F}_{\text {imag }}^{\pi}$ constrains the phase of the overlap $\left\langle 2\left|\hat{U}_{\pi}\right| 1\right\rangle$. All the conditions of the optimal inversion pulse [Eqs. (23)] are satisfied. Further, it can be shown that these performance functions [Eqs. (24) and (25)] are equivalent to obtaining the universal $180^{\circ}$ rotations explored by Kobzar et al. [42].

Previous work by Dunning et al. [8] defined the following fidelity for composite mirror pulses:

$$
\begin{aligned}
\mathcal{F}_{\text {square }}^{\pi} & =\left|\left\langle\psi_{F} \mid 2\right\rangle\right|^{2} \\
& =\left|c_{2}\right|^{2},
\end{aligned}
$$

where $c_{2}$ is the final excited state amplitude of the two-level system and $\left|\psi_{F}\right\rangle$ is the final state. The fidelity in Eq. (26) does not constrain the phase of the final state and yields pulses which are PP rotations between the ground and excited states. Since the mirror pulse in the Mach-Zehnder sequence is to be applied to atoms which ideally lie at a range of points on the equator of the Bloch sphere, we expect that pulses which maximize fidelity $\mathcal{F}_{\text {square }}^{\pi}$ will lead to poor interferometric contrast when averaging over an ensemble. Our approach is to use GRAPE to generate pulses which maximize a given mirror fidelity, $\mathcal{F}_{\text {real }}^{\pi}$, $\mathcal{F}_{\text {imag }}^{\pi}$, or $\mathcal{F}_{\text {square }}^{\pi}$ for ensembles with experimentally relevant ranges of detunings and coupling strengths. We then determine through simulation the effect of the cloud temperature alone upon the fringe contrast that these pulses could yield in an interferometric application.

\section{SYSTEM AND MODEL PARAMETERS}

Although our approach may be applied to any spin system, we evaluate it for a cold-atom light-pulse interferometer such as that in Ref. [8], wherein a thermal cloud of several million atoms of ${ }^{85} \mathrm{Rb}$ at a temperature of order $10-100 \mu \mathrm{K}$ is addressed by counterpropagating $780 \mathrm{~nm}$ laser beams tuned to a Raman transition between the hyperfine levels $\left|5^{2} S_{1 / 2}, F=2\right\rangle$ and $\left|5^{2} S_{1 / 2}, F=3\right\rangle$ (levels 1 and 2, respectively). The Raman beams are detuned far from single-photon resonance with the intermediate $\left|5^{2} P_{3 / 2}\right\rangle$ state, which may theoretically be adiabatically eliminated so that each atom can be described as an effective two-level system [5]. A description of our experimental arrangement is given elsewhere [8]. Our effective two-state system evolves under the Hamiltonian in Eq. (1), with $\Omega_{R}$ replaced by an effective two-photon Rabi frequency $\Omega_{1} \Omega_{2} /(2 \Delta)$, where $\Omega_{1}$ and $\Omega_{2}$ represent the coupling of each laser to levels 1 and 2 , respectively, and $\Delta$ represents the single photon detuning of both lasers from the intermediate level. The laser frequency becomes $\omega_{L}=\omega_{L 1}-\omega_{L 2}$, where $\omega_{L 1, L 2}$ are the frequencies of the counterpropagating Raman beams formed by lasers 1 and $2, \phi_{L}$ becomes an effective combined laser phase $\phi_{L 1}+\phi_{L 2}$, and $\mathbf{k}_{L}$ is the effective wave vector $\mathbf{k}_{L 1}-\mathbf{k}_{L 2}$.

In our atom cloud, there is Zeeman degeneracy over substates distinguished by the quantum number $m_{F}$, which 
TABLE I. Performance of GRAPE pulses compared with composite mirror pulses. Detuning tolerance is measured in units of the effective Rabi frequency $\Omega_{\text {eff }}$ and represents the range of detuning for which the final excited state probability is $>0.5$ and $>0.9$ after application to atoms in the ground state. The maximum phase response variation is taken over a range of $\pm \Omega_{\mathrm{eff}}$ in $\delta$. Optimization parameters are provided for GRAPE pulses including the offsets optimized for pulse-length and off-resonance errors and the number of offsets used. In each optimization, ensembles were weighted by eight additional detuning offsets near resonance. The best values for the standard composite sequences tested and GRAPE sequences are in bold.

\begin{tabular}{|c|c|c|c|c|c|c|}
\hline \multirow[b]{2}{*}{ Pulse } & \multirow{2}{*}{$\begin{array}{c}\text { Length } \\
\left(t / t_{\pi}\right)\end{array}$} & \multirow{2}{*}{\multicolumn{2}{|c|}{ Sequence $\theta_{\phi_{1}}^{(1)} \theta_{\phi_{2}}^{(2)} \theta_{\phi_{3}}^{(3)} \ldots$}} & \multicolumn{2}{|c|}{ Detuning tolerance $\left(\delta / \Omega_{\mathrm{eff}}\right)$} & \multirow{2}{*}{$\begin{array}{c}\operatorname{Max} \Delta \phi(S) \\
\quad \text { (radians) }\end{array}$} \\
\hline & & & & $>0.5$ & $>0.9$ & \\
\hline Rectangular $\pi$ & 1 & \multicolumn{2}{|c|}{$180_{0}$} & 1.597 & 0.645 & 0 \\
\hline Levitt [50] & 2 & \multicolumn{2}{|c|}{$90_{90} 180_{0} 90_{90}$} & 2.637 & 2.112 & 0.953 \\
\hline WALTZ [23] & 3 & \multicolumn{2}{|c|}{$90_{0} 180_{180} 270_{0}$} & 2.878 & 2.434 & 1.974 \\
\hline KNILL [46] & 5 & \multicolumn{2}{|c|}{$180_{240} 180_{210} 180_{300} 180_{210} 180_{240}$} & 2.082 & 1.693 & 0.528 \\
\hline CORPSE [21] & 4.333 & \multicolumn{2}{|c|}{$60_{0} 300_{180} 420_{0}$} & 1.438 & 1.004 & 2.717 \\
\hline SCROFULOUS [51] & 3 & \multicolumn{2}{|c|}{$180_{60} 180_{300} 180_{60}$} & 1.347 & 0.334 & 0.834 \\
\hline BB1 [52] & 5 & \multicolumn{2}{|c|}{$180_{104.5} 360_{313.4} 180_{104.5} 180_{0}$} & 1.685 & 1.106 & 1.778 \\
\hline \multicolumn{2}{|l|}{ GRAPE fidelity } & $\delta^{\text {off }}$ (number, range) & $\Omega_{R}{ }^{\text {off }}$ (number, rar & & & \\
\hline $\mathcal{F}_{\text {real }}^{\pi}$ Fig. 1 & 8 & $20, \pm 1.5 \Omega_{\mathrm{eff}}$ & $5, \pm 0.1 \Omega_{\mathrm{eff}}$ & 4.194 & 3.470 & 0.269 \\
\hline $\mathcal{F}_{\text {square }}^{\pi}$ & 8 & $20, \pm 1.5 \Omega_{\mathrm{eff}}$ & $5, \pm 0.1 \Omega_{\mathrm{eff}}$ & 3.968 & 3.376 & 1.562 \\
\hline $\mathcal{F}_{\text {imag }}^{\pi}$ & 8 & $20, \pm 1.5 \Omega_{\mathrm{eff}}$ & $5, \pm 0.1 \Omega_{\mathrm{eff}}$ & 3.904 & 3.259 & 0.137 \\
\hline $\mathcal{F}_{\text {real }}^{\pi}$ & 16 & $30, \pm 2 \Omega_{\mathrm{eff}}$ & $5, \pm 0.1 \Omega_{\mathrm{eff}}$ & 4.302 & 4.128 & 0.096 \\
\hline $\mathcal{F}_{\text {real }}^{\pi}$ & 32 & $40, \pm 2.5 \Omega_{\mathrm{eff}}$ & $5, \pm 0.1 \Omega_{\mathrm{eff}}$ & 5.513 & 5.109 & 0.216 \\
\hline
\end{tabular}

give rise to multiple coupling strengths, and both the laser intensity and residual magnetic field vary across the atom cloud. The counterpropagating Raman beams give the interaction a Doppler sensitivity, which we use elsewhere for velocimetry and inertial measurement [43]. Different atoms thus see different coupling strengths and Doppler, Zeeman, and ac Stark shifts according to their internal and external states. Both pulse-length and off-resonance errors are present in our system. For an experimentally measured Rabi frequency of $\Omega_{\text {eff }} \approx 2 \pi \times 360 \mathrm{kHz}$ we find coupling strength variations of approximately $0.3 \Omega_{\text {eff }}$ and off-resonance errors due to a Gaussian velocity distribution with a full width at half maximum of approximately $1.5 \Omega_{\text {eff }}$ in a $\sim 80 \mu \mathrm{K}$ cloud [44].

\section{RESULTS}

All pulse optimizations were carried out using GRAPE and Spinach, constraining the effective Rabi frequency to correspond to a limited laser power and fixed single-photon detuning, and with a discretization time step of $100 \mathrm{~ns}$. Optimizations were carried out for ensembles with various ranges of detunings and coupling strengths (see Table I), using fidelities $\mathcal{F}_{\text {real }}^{\pi}, \mathcal{F}_{\text {imag }}^{\pi}$, and $\mathcal{F}_{\text {square }}^{\pi}$. Longer pulses can excite atoms from the ground to excited states for larger detunings than shorter pulses. Mirror pulses are compared based on their ability to transfer atoms from state to state and the magnitude of the atomic phase variations over the simulated ensemble of atoms. The mirror pulse is characterized by a time-varying phase, initially set to be flat, and optimization was continued until convergence. Different initial pulse shapes were often found to lead to the same optimal pulse, and pulses often exhibited symmetry about the midpoint, a phenomenon also seen in NMR $[41,42,45]$. We begin by presenting the results obtained by considering a general ensemble of pulselength and off-resonance errors in our optimization, before applying our chosen optimal control method to an ensemble representing the experimental inhomogeneities present in a particular Raman polarization arrangement.

\section{A. Mirror pulse optimization}

Figure 1 shows the phase profile of a constant-amplitude mirror pulse obtained by maximizing $\mathcal{F}_{\text {real }}^{\pi}$ subject to a constrained duration of $20 \mu \mathrm{s}$, for a detuning range of $\pm 1.5 \Omega_{\mathrm{eff}}$ and a power range of \pm 0.1 in $\Omega_{\text {eff. }}$. If the duration of the pulse and the ensemble detuning range are increased, further variations in phase appear symmetrically around the center of the pulse shape. In practice, a balance must be struck between pulse duration and performance, dependent on the required application and capabilities of the experimental apparatus to

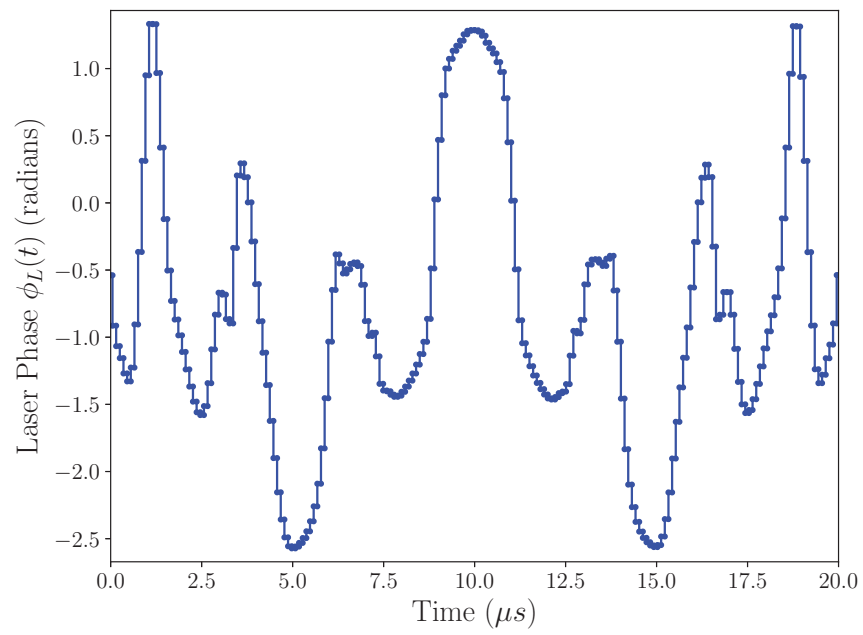

FIG. 1. Phase profile $\phi_{L}(t)$ for GRAPE inversion pulse optimizing $\mathcal{F}_{\text {real }}^{\pi}$ subject to constrained total duration of $20 \mu$ s and fixed effective Rabi frequency of $2 \pi \times 200 \mathrm{kHz}$. The fidelity after 100 iterations was 0.99 . 


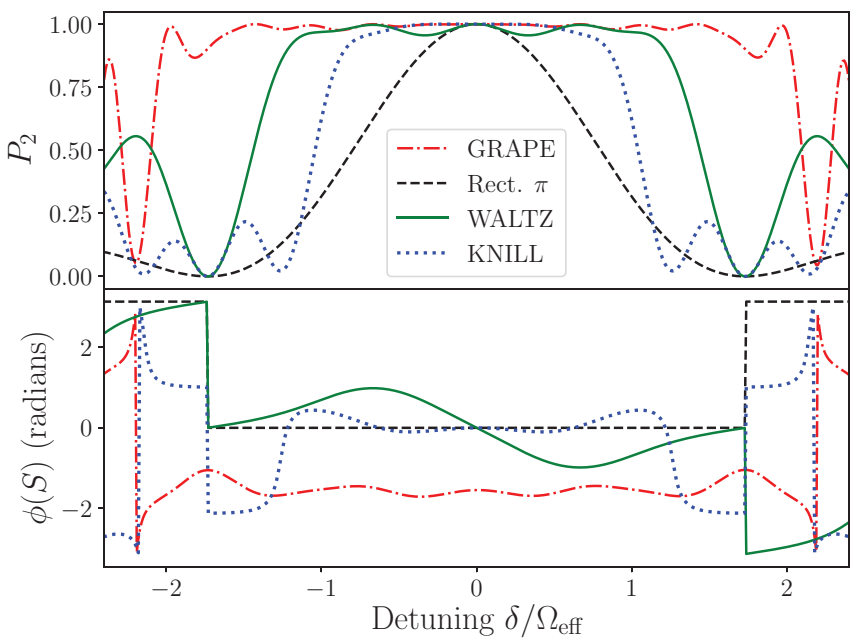

FIG. 2. Excited state probability and phase profiles plotted against detuning for various composite inversion pulses and GRAPE pulse of Fig. 1. The simulation assumes a single Rabi frequency of $2 \pi \times 200 \mathrm{kHz}$.

produce complex waveforms. Table I summarizes the performance of composite mirror pulses and GRAPE optimizations carried out for different ensembles and fidelities.

The response purely as a function of the detuning from resonance is shown in Fig. 2. The detuning here would be experimentally due to Doppler and Zeeman shifts. Both the excited state probability after application of the pulse to atoms in the ground state and the phase response of the $S$ element of the propagator are shown. GRAPE maintains a transition probability $>0.976$ over the optimized range of offsets $\left( \pm 1.5 \Omega_{\text {eff }}\right)$. For comparison, we show the corresponding results for the rectangular $\pi$ pulse and for WALTZ and KNILL pulses $[23,46]$ of the same intensity. The WALTZ pulse is highly robust to detunings but is designed as a "point-to-point" operation for a particular starting state and hence shows large variations in the atomic phase as the detuning is varied; the GRAPE pulse, in contrast, shows smaller phase variations over the range of detunings. The conventional $\pi$ pulse shows an ideal, flat phase response but very narrow range of detunings over which it is efficient.

GRAPE can be used to optimize a mirror pulse for an experimental range of off-resonance errors and the pulse-length errors which arise from the degenerate Zeeman sublevels in the $\sigma^{+}-\sigma^{+}$Raman polarization arrangement. We simulate the pulse profile of Fig. 1 using a model which accounts for the ac Stark shift, $m_{F}$ levels, measured atomic momentum distribution, and experimental parameters in the $\sigma^{+}-\sigma^{+}$polarization arrangement for a cloud with temperature $\sim 80 \mu \mathrm{K}$ (see Fig. 3). Such an arrangement has large off-resonance errors and is therefore a good choice to compare pulse performance. The numerical model used in this simulation was shown to have good agreement with experiment $[8,44]$. In this simulation, the final excited state population is calculated numerically and averaged over a range of atomic momenta from the measured distribution, and the five Zeeman sublevels. The GRAPE simulations show an improvement in peak fidelity by a factor of 1.2 compared with WALTZ, and a factor of 2 compared with the rectangular $\pi$ pulse. As the laser detuning

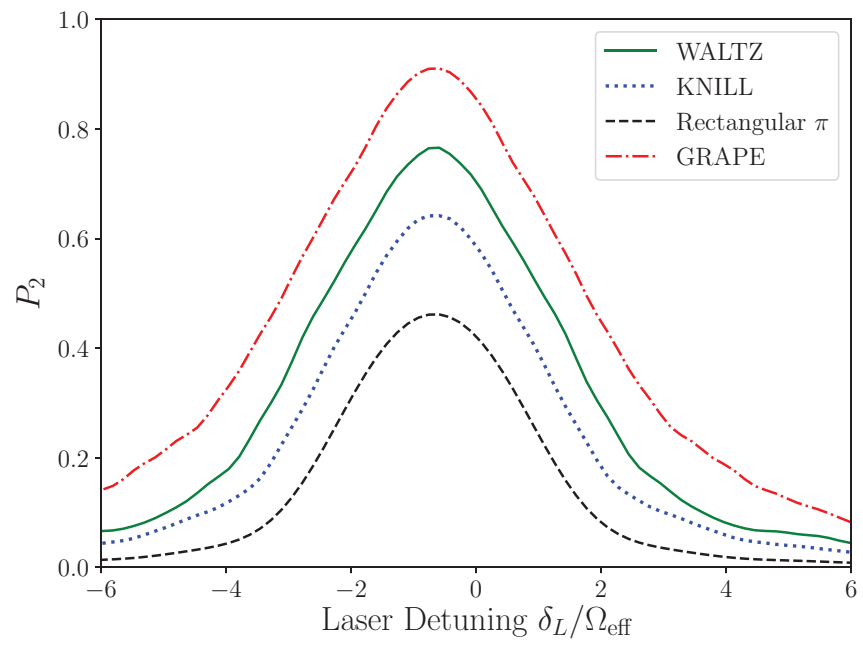

FIG. 3. Simulation of inversion operation for multiple composite pulses and the GRAPE pulse of Fig. 1 in a $\sigma^{+}-\sigma^{+}$Raman arrangement, as the laser detuning $\delta_{L}$ (defined as $\omega_{12}-\omega_{L}$ ) is scanned. This simulation uses an effective Rabi frequency of $2 \pi \times 360 \mathrm{kHz}$, and accounts for both the coupling strengths of $5 m_{\mathrm{F}}$ sublevels and the Stark shift. Both the simulation parameters and model are described elsewhere $[8,44]$. The offset in peak is due to the Stark shift, and the magnitude of the peak gives an indication of a pulse's ability to excite atoms across the momentum distribution for all $m_{F}$ sublevels, which we assume are equally populated.

is scanned, the peak excited state population depends on how well a mirror pulse can excite atoms from the ground to excited state for the distribution of coupling strengths and velocity classes in the ensemble. Figure 3 demonstrates GRAPE's ability to compensate for the off-resonance and pulse-length errors present in this system. Contour plots (Fig. 4) demonstrate the superior robustness to both error classes offered by our chosen optimal control method.

\section{B. Simulated interferometric contrast}

The temperature-dependent contrast resulting from the Mach-Zehnder interferometer sequence was determined following the procedure outlined by Luo et al. [14] and integrating over Maxwell-Boltzmann velocity distributions with temperatures in the range $10^{-1}$ to $10^{3} \mu \mathrm{K}$. The results are shown in Fig. 5. A uniform pulse intensity and single coupling strength, with no $m_{F}$-dependent inhomogeneity, were assumed throughout. The beam-splitter and recombiner pulses were taken to be rectangular $\pi / 2$ pulses with a fixed effective Rabi frequency of $2 \pi \times 200 \mathrm{kHz}$ and hence limit the achievable fringe contrast as shown by their combination with a "perfect $\pi$ " mirror pulse that is taken to perform an ideal rotation for all atoms. With a realistic mirror formed by a rectangular $\pi$ pulse with the same Rabi frequency as the beam splitter and recombiner, the interferometer contrast is limited by the imperfections in the rectangular $\pi$ pulse to around 0.8 at a temperature of $20 \mu \mathrm{K}$.

The GRAPE pulse of Fig. 1 offers an improvement in fringe contrast by a factor of 1.76 over that with the rectangular $\pi$ pulse at $100 \mu \mathrm{K}$, and approaches the fidelity predicted with a perfect $\pi$ pulse. This limit should be approached more closely as the GRAPE pulse duration is increased, as longer pulses can 


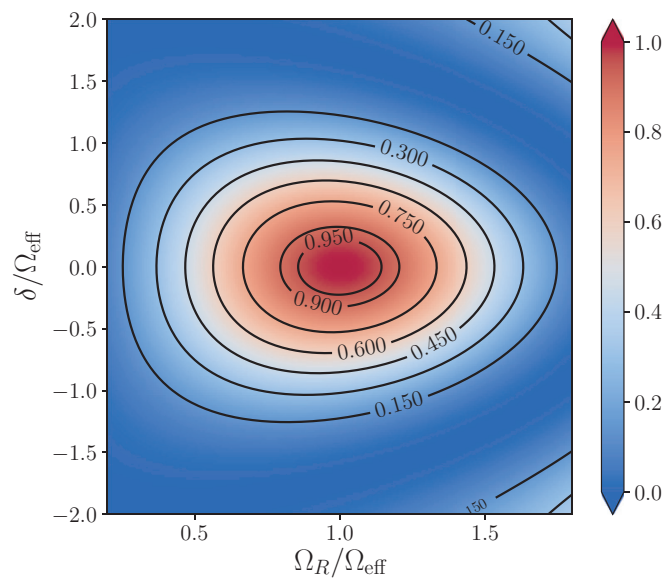

(a)

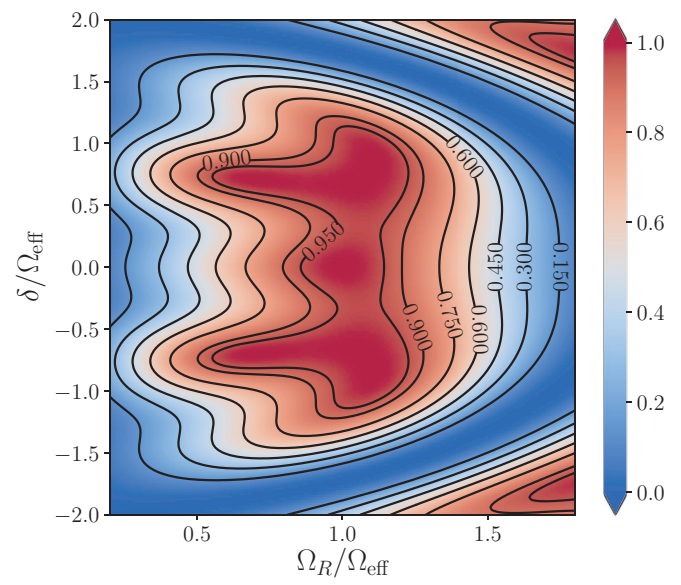

(c)

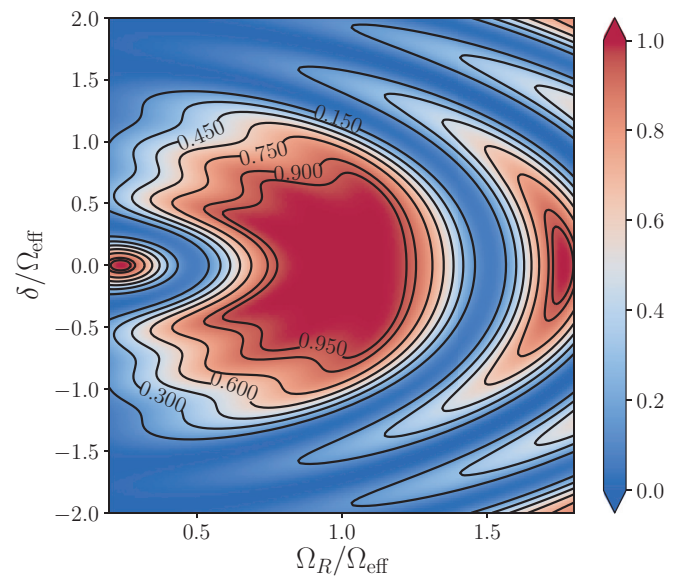

(b)

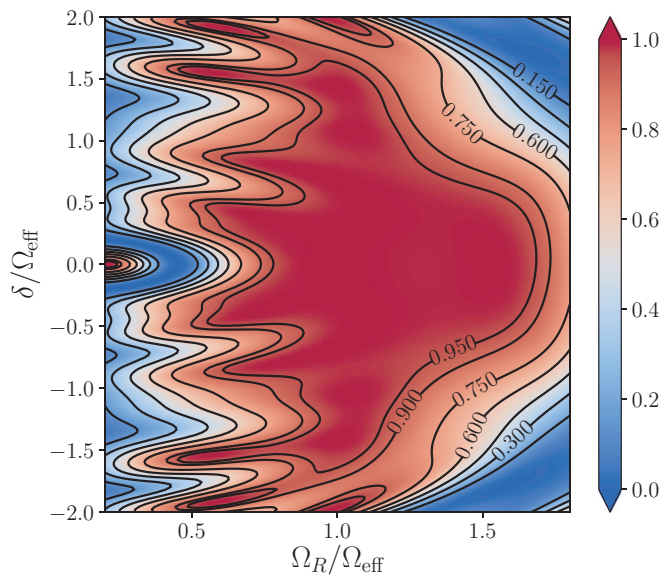

(d)

FIG. 4. Final excited state population shown for a range of off-resonance and pulse-length errors for different mirror pulses: (a) rectangular $\pi$ pulse; (b) KNILL pulse; (c) WALTZ pulse; (d) GRAPE pulse from Fig. 1. The simulations were performed at the same effective Rabi frequency of $2 \pi \times 200 \mathrm{kHz}$. The GRAPE mirror pulse demonstrates a clear improvement in robustness to variations in detuning and pulse amplitude. The contours are at $0.15,0.3,0.45,0.6,0.75,0.9$, and 0.95 .

compensate for a greater range of off-resonance errors. As noted by Luo et al. [14,47], the WALTZ pulse performs badly due to its nonuniform phase response, as it was designed as a PP pulse whereas the mirror operation must have high fidelity irrespective of the starting point on the equator of the Bloch sphere. The PP $\mathcal{F}_{\text {square }}^{\pi}$ GRAPE pulse also performs poorly, as expected. We find the KNILL pulse, designed as a universal $180^{\circ}$ rotation, offers a slight improvement over the sequence of rectangular pulses for the simulated range of temperatures but is outperformed by GRAPE at all temperatures.

\section{DISCUSSION}

Tolerance of "pulse-length" and "off-resonance" errors is essential for the pulse operations in atom interferometers, where a range of velocities, beam intensities, and Zeeman substates may be encountered. We have used the optimal control technique of gradient ascent pulse engineering (GRAPE) to obtain robust "mirror" pulses, tailored to accommodate the inhomogeneities found in cold-atom matterwave interferometers, and find such pulses to outperform all the other composite pulses that we have tested. By using a numerical model which has been shown to agree well with experiment [8], we have simulated the performance of an atom interferometer for the $\sigma^{+}-\sigma^{+}$polarization Raman arrangement within a $\sim 80 \mu \mathrm{K}$ cloud of atomic rubidium subject to realistic intensity inhomogeneities and Zeeman substate distributions, and we find that our GRAPE pulses show a peak fidelity twice that obtained with a basic rectangular $\pi$ pulse, and 1.2 times that achieved using the WALTZ sequence, with significantly greater tolerance of variations in the atom velocity.

The improved fidelity should allow improvements in the sensitivity of interferometric measurements by permitting greater use of augmentation pulses for large momentum transfer interferometers [12], while the tolerance to atom velocity variations will lower measurement noise by allowing the use of warmer atom clouds, and hence higher atom numbers, without incurring the losses of further cooling or filtering. Our GRAPE pulses should provide, for example, transfer efficiencies above 0.9 for a detuning range 1.4 times that tolerated by the WALTZ sequence, which was otherwise the best pulse tested for this system [8]. 


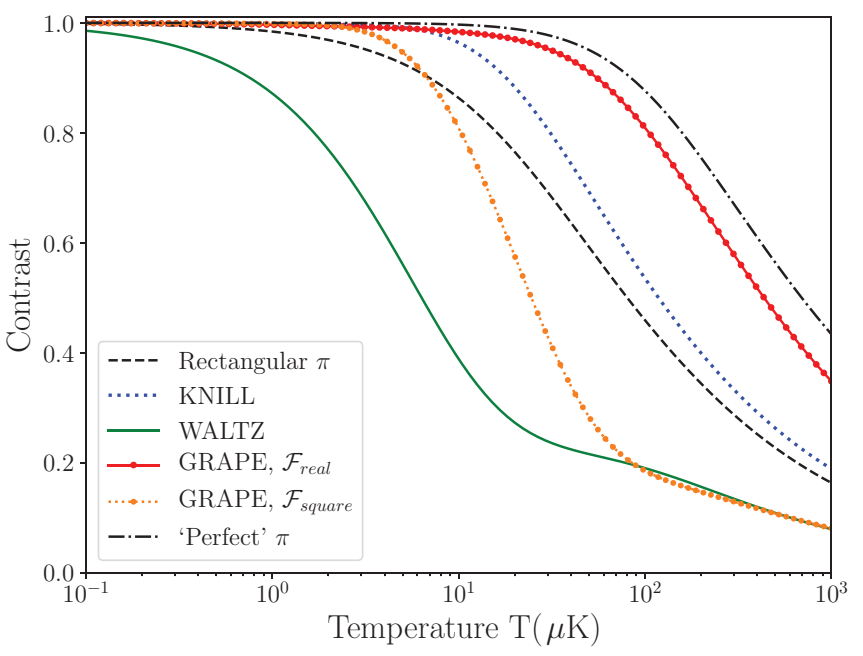

FIG. 5. Contrast after Mach-Zehnder sequence integrated over thermal distributions of ${ }^{85} \mathrm{Rb}$ atoms with various temperatures. The beam splitters in each case are rectangular $\pi / 2$ pulses. There is a large increase in contrast due to the GRAPE UR mirror pulse in Fig. 1, which improves the rectangular $\pi$ pulse by a factor of 1.76 at $100 \mu \mathrm{K}$, approaching the theoretical limit of a sequence with a "perfect" $\pi$ pulse resonant for all atoms in the sample. Comparisons with different sequences are made at the same Rabi frequency of $2 \pi \times 200 \mathrm{kHz}$.

Replacing the basic rectangular $\pi$ pulse with a GRAPE mirror in a Mach-Zehnder arrangement at $100 \mu \mathrm{K}$ is sufficient to improve the simulated interferometer contrast by a factor of 1.76 , or to achieve the same contrast as a basic $\pi$ pulse for a $15 \mu \mathrm{K}$ atom cloud. At higher atom temperatures, we see that the interferometer fidelity is limited principally by the fidelity of the beam-splitter operations.

Our optimal control approach depends upon an appropriate choice of the measure of performance. We find that those used for broadband UR $180^{\circ}$ pulses, such as $\mathcal{F}_{\text {real }}^{\pi}$ [Eq. (24)] and equivalents considered by Kobzar et al. [42], are able to preserve the interferometric phase and increase contrast, and when our optimization is carried out for small detuning ranges we produce similar pulse shapes. Measures of performance more suitable for PP operations, such as $\mathcal{F}_{\text {square }}^{\pi}$ [Eq. (26)], conversely yield lower interferometer contrast, as does the WALTZ point-to-point composite pulse.

While GRAPE should also be applicable to the design of beam-splitter pulses, we expect that a more fruitful approach will be to consider symmetries in the Mach-Zehnder sequence and compensate in the second beam-splitter operation for errors introduced in the first, so as to optimize the interferometer as a whole. Such cooperative pulse optimization was investigated by Braun et al. [48,49] for Ramsey-style experiments in NMR and allows greater freedom in the optimization as individual beam-splitter pulses are permitted imperfections provided that they are canceled elsewhere in the interferometer sequence. This should allow shorter pulse sequences, desirable for interferometric sensors operating in dynamic environments [28] and is attractive for optimization of the $\pi / 2-\pi-\pi / 2$ sequence used for inertial sensing applications. Nonetheless, we expect the mirror optimization described here to suffice for a large contrast improvement in many current configurations.

Our future work will involve experimental demonstration of GRAPE mirror pulses in our experiment and analysis of how features present in pulse profiles from optimal control methods allow for error compensation. This will involve characterizing the dynamics and evolution of atomic trajectories on the Bloch sphere.

\section{ACKNOWLEDGMENTS}

The authors are grateful for the advice provided by David Elcock and Matt Himsworth. This work was supported by the EPSRC through the UK Quantum Technology Hub for Sensors \& Metrology under grant EP/M013294/1, the Centre for Doctoral Training in Next Generation Computational Modelling under grant EP/L015382/, and by Defence Science and Technology Laboratory, UK under Grants No. DSTLX-1000091758 and No. DSTLX-1000097855.
[1] M. Kasevich and S. Chu, Phys. Rev. Lett. 67, 181 (1991).

[2] T. L. Gustavson, P. Bouyer, and M. A. Kasevich, Phys. Rev. Lett. 78, 2046 (1997).

[3] M. De Angelis, A. Bertoldi, L. Cacciapuoti, A. Giorgini, G. Lamporesi, M. Prevedelli, G. Saccorotti, F. Sorrentino, and G. M. Tino, Meas. Sci. Technol. 20, 022001 (2009).

[4] B. Barrett, R. Geiger, I. Dutta, M. Meunier, B. Canuel, A. Gauguet, P. Bouyer, and A. Landragin, C. R. Phys. 15, 875 (2014).

[5] Atom Interferometry, edited by P. Berman (Academic Press, Cambridge, MA, 1997), p. 363.

[6] J. M. McGuirk, M. J. Snadden, and M. A. Kasevich, Phys. Rev. Lett. 85, 4498 (2000).

[7] R. U. Islam, A. H. Khosa, and F. Saif, J. Phys. B 41, 035505 (2008).

[8] A. Dunning, R. Gregory, J. Bateman, N. Cooper, M. Himsworth, J. A. Jones, and T. Freegarde, Phys. Rev. A 90, 033608 (2014).

[9] M. Kasevich, D. S. Weiss, E. Riis, K. Moler, S. Kasapi, and S. Chu, Phys. Rev. Lett. 66, 2297 (1991).
[10] J. M. McGuirk, G. T. Foster, J. B. Fixler, M. J. Snadden, and M. A. Kasevich, Phys. Rev. A 65, 033608 (2002).

[11] S. S. Szigeti, J. E. Debs, J. J. Hope, N. P. Robins, and J. D. Close, New J. Phys. 14, 023009 (2012).

[12] D. L. Butts, K. Kotru, J. M. Kinast, A. M. Radojevic, B. P. Timmons, and R. E. Stoner, J. Opt. Soc. Am. B 30, 922 (2013).

[13] R. Freeman, Prog. Nucl. Magn. Reson. Spectrosc. 32, 59 (1998).

[14] Y. Luo, S. Yan, Q. Hu, A. Jia, C. Wei, and J. Yang, Eur. Phys. J. D 70, 262 (2016).

[15] B. Fang, N. Mielec, D. Savoie, M. Altorio, A. Landragin, and R. Geiger, New J. Phys. 20, 023020 (2018).

[16] J. Baum, R. Tycko, and A. Pines, Phys. Rev. A 32, 3435 (1985).

[17] T. Kovachy, S. W. Chiow, and M. A. Kasevich, Phys. Rev. A 86, 011606 (2012).

[18] J. Bateman and T. Freegarde, Phys. Rev. A 76, 013416 (2007).

[19] M. H. Levitt and R. Freeman, J. Magn. Reson. (1969) 43, 502 (1981).

[20] M. H. Levitt and R. R. Ernst, J. Magn. Reson. (1969) 55, 247 (1983). 
[21] H. K. Cummins and J. A. Jones, New J. Phys. 2, 6 (2000).

[22] P. Berg, S. Abend, G. Tackmann, C. Schubert, E. Giese, W. P. Schleich, F. A. Narducci, W. Ertmer, and E. M. Rasel, Phys. Rev. Lett. 114, 063002 (2015).

[23] A. J. Shaka, J. Keeler, T. Frenkiel, and R. Freeman, J. Magn. Reson. (1969) 52, 335 (1983).

[24] N. Khaneja, T. Reiss, C. Kehlet, T. Schulte-Herbrüggen, and S. J. Glaser, J. Magn. Reson. 172, 296 (2005).

[25] H. J. Hogben, M. Krzystyniak, G. T. P. Charnock, P. J. Hore, and I. Kuprov, J. Magn. Reson. 208, 179 (2011).

[26] B. W. Shore, Manipulating Quantum Structures Using Laser Pulses (Cambridge University Press, New York, 2011).

[27] D. J. Tannor, Introduction to Quantum Mechanics: A TimeDependent Perspective (University Science Books, Sausalito, CA, 2007).

[28] R. Stoner, D. Butts, J. Kinast, and B. Timmons, J. Opt. Soc. Am. B 28, 2418 (2011).

[29] T. E. Skinner, T. O. Reiss, B. Luy, N. Khaneja, and S. J. Glaser, J. Magn. Reson. 163, 8 (2003).

[30] S. van Frank, A. Negretti, T. Berrada, R. Bücker, S. Montangero, J.-F. Schaff, T. Schumm, T. Calarco, and J. Schmiedmayer, Nat. Commun. 5, 4009 (2014).

[31] G. Jäger, D. M. Reich, M. H. Goerz, C. P. Koch, and U. Hohenester, Phys. Rev. A 90, 033628 (2014).

[32] P. B. Wigley, P. J. Everitt, A. van den Hengel, J. W. Bastian, M. A. Sooriyabandara, G. D. McDonald, K. S. Hardman, C. D. Quinlivan, P. Manju, C. C. N. Kuhn, I. R. Petersen, A. N. Luiten, J. J. Hope, N. P. Robins, and M. R. Hush, Sci. Rep. 6, 25890 (2016).

[33] C. P. Koch, J. P. Palao, R. Kosloff, and F. Masnou-Seeuws, Phys. Rev. A 70, 013402 (2004).

[34] A. D. Tranter, H. J. Slatyer, M. R. Hush, A. C. Leung, J. L. Everett, K. V. Paul, P. Vernaz-Gris, P. K. Lam, B. C. Buchler, and G. T. Campbell, arXiv:1805.00654.

[35] T. Nöbauer, A. Angerer, B. Bartels, M. Trupke, S. Rotter, J. Schmiedmayer, F. Mintert, and J. Majer, Phys. Rev. Lett. 115, 190801 (2015).
[36] F. F. Floether, P. De Fouquieres, and S. G. Schirmer, New J. Phys. 14, 073023 (2012).

[37] D. L. Goodwin and I. Kuprov, J. Chem. Phys. 143, 084113 (2015).

[38] D. L. Goodwin and I. Kuprov, J. Chem. Phys. 144, 204107 (2016).

[39] P. De Fouquieres, S. G. Schirmer, S. J. Glaser, and I. Kuprov, J. Magn. Reson. 212, 412 (2011).

[40] M. H. Levitt, Spin Dynamics: Basic Principles of Nuclear Magnetic Resonance, 2nd ed. (John Wiley and Sons, Chichester, UK, 2008).

[41] K. Kobzar, T. E. Skinner, N. Khaneja, S. J. Glaser, and B. Luy, J. Magn. Reson. 170, 236 (2004).

[42] K. Kobzar, S. Ehni, T. E. Skinner, S. J. Glaser, and B. Luy, J. Magn. Reson. 225, 142 (2012).

[43] M. Carey, M. Belal, M. Himsworth, J. Bateman, and T. Freegarde, J. Mod. Opt. 65, 657 (2017).

[44] A. Dunning, Coherent atomic manipulation and cooling using composite optical pulse sequences, Ph.D. thesis, University of Southampton, UK (2014).

[45] K. Kobzar, T. E. Skinner, N. Khaneja, S. J. Glaser, and B. Luy, J. Magn. Reson. 194, 58 (2008).

[46] C. A. Ryan, J. S. Hodges, and D. G. Cory, Phys. Rev. Lett. 105, 200402 (2010).

[47] Y. Luo, S. Yan, J. Yang, Q. Hu, A. Jia, C. Wei, and G. Wang, in 2015 11th Conf. on Lasers and Electro-Optics Pacific Rim, CLEO-PR 2015 (IEEE, Busan, South Korea, 2016), Vol. 2, p. 2.

[48] M. Braun and S. J. Glaser, J. Magn. Reson. 207, 114 (2010).

[49] M. Braun and S. J. Glaser, New J. Phys. 16, 115002 (2014).

[50] M. H. Levitt and R. Freeman, J. Magn. Reson. (1969) 43, 65 (1981).

[51] H. K. Cummins, G. Llewellyn, and J. A. Jones, Phys. Rev. A 67, 042308 (2003).

[52] S. Wimperis, J. Magn. Reson., Ser. A 109, 221 (1994).

Correction: Minor errors in Eqs. (8) and (22) have been fixed. 